EPV133/\#555 MINIMALLY INVASIVE APPROACH IN ENDOMETRIAL CANCER WITH LOWER UTERINE SEGMENT INVOLVEMENT IN $\geq$ STAGE II: IS IT SAFE?

${ }^{1} \mathrm{~T}$ Perri, ${ }^{2} \mathrm{~L}$ Kogan ${ }^{*},{ }^{3} \mathrm{~L}$ Helpman, ${ }^{4} \mathrm{R}$ Eitan, ${ }^{5} \mathrm{Z}$ Vaknin, ${ }^{6} \mathrm{O}$ Lavie, ${ }^{7} \mathrm{~A}$ Ben Arie, ${ }^{8} \mathrm{~A}$ Amit, ${ }^{9} \mathrm{~T}$ Levy, ${ }^{10} \mathrm{~A}$ Namazov, ${ }^{11}$ | Ben Shachar, ${ }^{12}$ | Atlas, ${ }^{13}$ I Bruchim, ${ }^{1} \mathrm{G}$ Levin, ${ }^{14} \mathrm{O}$ Gemer. ${ }^{1}$ Hadassah Medical Center, Gynecologic Oncology, Jerusalem, Israel; ${ }^{2}$ Hadassah, Gynecologic Oncology, Jerusalem, Israel; ${ }^{3}$ Cheba medical center, Gynecologic Oncology, Ramat Gan, Israel; ${ }^{4}$ Rabin Medical Center, Gynecology, Petah Tikva, Israel; ${ }^{5}$ Assaf Haroffe Medical Center, Sackler School of Medicine, Gynecology, Zrifin, Israel; ${ }^{6}$ Carmel Medical Center, Obstetrics and Gynecology, Haifa, Israel; '72. Kaplan Medical Center, Hebrew University, Gynecology, Rehovot, Israel; ${ }^{8}$ Rambam, Gymecologic Oncology, Haifa, Israel; ${ }^{9} 8$. Wolfson Medical Center, Holon, Sackler Faculty of Medicine, Tel Aviv University, Gynecology, Tel Aviv, Israel; ${ }^{10}$ Ahmet, Gynecology, Ashkelon, Israel; ${ }^{11}$ Zlv, Gynecologic Oncology, Zefat, Israel; ${ }^{12}$ Poriah, Gynecologic Oncology, Tiberia, Israel; ${ }^{13}$ Hillel Yafe, Gynecology, Hadera, Israel; ${ }^{14}$ Barzilai Medical Center, Gynecology, Ashkelon, Israel

10.1136/ijgc-2021-IGCS.203

Objectives To compare survival outcomes between women with stages II-IV endometrial carcinoma (EC) with lower uterine segment involvement (LUSI), staged by minimally invasive surgery (MIS) and those staged by laparotomy.

Methods A retrospective multi-center cohort study of nine gynecologic-oncology centers. Univariate analysis, Kaplan-Meier survival and Cox proportional hazard models analysis were performed to compare women surgically staged by MIS and those operated by laparotomy in different stages and histology of EC.

Results Over a median follow-up period of 3 years (interquartile range, 1.5-6 years) 212 women were included, 68 $(32.1 \%)$ were surgically staged by MIS. Stages of disease among the study cohort were stages II, III and IV, 32.1\%, $51.9 \%$, and $16.0 \%$, respectively. Stage distribution did not vary between MIS and laparotomy groups $(p=0.144)$. Highgrade histology was less common in MIS group $(44.1 \%$ vs. $67.4 \%, \mathrm{p}<0.001)$. Adjuvant radiation and chemotherapy rates were comparable. Recurrence (local and distal) rate did not differ between groups $(44.1 \%$ MIS vs. $31.9 \%$ laparotomy, $\mathrm{p}=0.084)$. Local recurrence rate was higher in MIS group $(32.4 \%$ vs. $18.1 \%, p=0.023)$. Overall survival and local recurrence-free survival were similar in both groups (log rank test $\mathrm{p}=0.08, \mathrm{p}=0.33$, respectively). In Cox regression model adjusting for age, comorbidities, tumor grade, disease stage and adjuvant therapy, route of surgery (MIS vs. laparotomy) was not associated with overall survival $(p=0.169)$ or local recurrence $(p=0.296)$.

Conclusions In women with stage II-IV EC with LUSI, MIS was associated with higher local recurrence rate, yet overall survival was comparable between patients with MIS and laparotomy, regardless of adjuvant therapy.

\section{EPV134/\#557 THE STUDY OF SOME CLINICO-GENETIC CHARACTERISTICS IN PREGNANT WOMEN WITH UTERINE LEIOMYOMAS}

1) Ungiadze, ${ }^{1} \mathrm{~N}$ Zamtaradze, ${ }^{2} \mathrm{~K}$ Samnidze, ${ }^{3} \mathrm{D}$ Ungiadze*, ${ }^{1}$ I Nakashidze. ${ }^{1}$ Batumi Shota Rustaveli State University, Department of Clinical Medicine, Batumi, Georgia; ${ }^{2}$ Medina, Department of Gynecology, Batumi, Georgia; ${ }^{3}$ Tbilisi State Medical University, Department of Clinical Medicine, Tbilisi, Georgia

10.1136/ijgc-2021-IGCS.204
Objectives Uterine leiomyomas (ULs) are common among pregnant women. Besides numerous investigations, the relationship between ULs and adverse pregnancy outcome is not clearly understood until now. Notably, the majority of fibroids do not change their size during pregnancy, but one-third may grow in the first trimester, also may cause multiple complications

Methods We evaluated some clinical variables, including the following: the patient age, the size and number of uterine fibroids, serum levels of Anti-TPO, Ft4, TSH, Glucose, Antiphospholipid Antibodies and Ferritin. Also, we investigated the fetus risk for trisomies of 13, 18, and 21 chromosomes. In present study, we included 20 pregnant women (10 with leiomyomas (ages - 37 $\pm 2,334$ ) and 10 without leiomyomas (ages - $38 \pm 3,44)) . \quad \mathrm{P}<0.05$ was regarded as statistically significant.

Results Our study suggested that ULs are associated with hypothyroidism in pregnant women with ULs. Notably, our studies show that all fetuses are non-affected, according to trisomies.

Conclusions In conclusion, we have thought that the hypothyroidism may some role in ULs.

\section{EPV135/\#596 CLINICAL AND PATHOLOGICAL FEATURES OF ENDOMETRIAL CANCER PATIENTS WITH DNA MISMATCH REPAIR DEFICIENCY TREATED AT A BRAZILIAN CANCER HOSPITAL}

E Mayerhoff*, C Anton, M Wagner, J Carvalho. Instituto do Câncer do Estado de São Paulo, Ginecologia E Obstetrícia, Sao Paulo, Brazil

\subsection{6/ijgc-2021-IGCS.205}

Objectives Identification of DNA mismatch repair (MMR) deficiency has been proposed as a screening strategy for Lynch Syndrome (LS) in endometrial cancer (EC) patients and is useful in predicting tumor sensitivity for immune checkpoint blockade therapies. The proportion of EC with MMR deficiency is reported to be $26 \%$, and around 3\% of EC may be attributed to LS. The present study aims to identify clinicopathological features of EC patients tested for tumoral MMR expression in a Brazilian cancer center.

Methods 479 patients treated for EC from 2010 through 2020 at Instituto do Câncer do Estado de São Paulo (ICESP) had their tumors analyzed by immunohistochemistry for MLH-1, MSH-2, MSH-6 and PMS-2. Clinical and pathological information from these cases were retrieved using a REDCap database and statistics were calculated using the SPSS software.

Results From the 479 cases analyzed, 453 resulted in conclusive immunostainings for MMR enzymes: 305 (67\%) were MMR proficient (pMMR) and 148 (33\%) were MMR deficient (dMMR). Results comparing the two groups are shown in table 1.

Conclusions In this population, dMMR EC had a higher prevalence than previously reported. Detection of germline mutation is necessary to investigate whether LS is more prevalent. Clinical aspects did not differ between groups. Lymphvascular space invasion was more frequent in the tumors of the dMMR group, whereas aberrant p53 immunostaining was 